\title{
Computational healthcare: Present and future perspectives (Review)
}

\author{
AYUMU ASAI ${ }^{1-3}$, MASAMITSU KONNO $^{1}$, MASATERU TANIGUCHI $^{3}$, ANDREA VECCHIONE $^{4}$ and HIDESHI ISHII $^{1}$ \\ ${ }^{1}$ Center of Medical Innovation and Translational Research, Department of Medical Data Science, Graduate School of Medicine, \\ Osaka University, Suita, Osaka 565-0871; ${ }^{2}$ Artificial Intelligence Research Center; ${ }^{3}$ The Institute of Scientific and \\ Industrial Research, Osaka University, Ibaraki, Osaka 567-0047, Japan; ${ }^{4}$ Department of Clinical and Molecular Medicine, \\ University of Rome 'Sapienza', Santo Andrea Hospital, I-1035-00189 Rome, Italy
}

Received January 14, 2021; Accepted July 19, 2021

DOI: $10.3892 /$ etm.2021.10786

\begin{abstract}
Artificial intelligence (AI) has been developed through repeated new discoveries since around 1960. The use of AI is now becoming widespread within society and our daily lives. AI is also being introduced into healthcare, such as medicine and drug development; however, it is currently biased towards specific domains. The present review traces the history of the development of various AI-based applications in healthcare and compares AI-based healthcare with conventional healthcare to show the future prospects for this type of care. Knowledge of the past and present development of AI-based applications would be useful for the future utilization of novel AI approaches in healthcare.
\end{abstract}

\section{Contents}

1. Introduction

2. Historical view of the clinical application of computational support

3. Current AI applied in medicine

4. AI in drug development as a foundation for drug therapy

5. Perspectives for AI-based medicine and drug development

6. Conclusion

Correspondence to: Professor Hideshi Ishii, Center of Medical Innovation and Translational Research, Department of Medical Data Science, Graduate School of Medicine, Osaka University, 2-2 Yamadaoka, Suita, Osaka 565-0871, Japan

E-mail: hishii@gesurg.med.osaka-u.ac.jp

Key words: computational medicine, artificial intelligence, artificial intelligence-based application, diagnosis, treatment, follow-up, drug development

\section{Introduction}

'Big data', large datasets that are difficult to record, store and analyze with conventional data management systems, has been accumulating in various fields in recent years with regard to the development of communication and sensor technology. The advances in technology regarding big data have emerged that the use of big data is expected to create new avenues of research. However, the overall trend of big data is difficult to understand based on general information processing by humans; thus, information processing by artificial intelligence (AI) has also attracted attention (1). In general, industries have succeeded in improving sales and work efficiency and decreasing costs using big data and AI (2).

In healthcare, the creation of new knowledge and the improvement in diagnostic and therapeutic outcomes are expected through the utilization of big data pertaining to life science information and medical data (3). In fact, the implementation of AI in healthcare has been actively investigated; however, it has not been used in a widespread manner due to a number of problems (4).

The present review looks back at the history of AI and AI-based applications, compares the advantages and the issues of conventional healthcare and AI-based healthcare, and considers the future development of AI-based applications.

\section{Historical view of the clinical application of computational support}

In the 1950s, McCarthy et al (5) proposed AI as a prediction machine (hardware or software that exhibits behavior which appears intelligence by predicting associations between variables). Samuel (6) developed machine learning in 1959, which triggered the first AI boom (Fig. 1). In this period, the discrimination of cells in microscopic images started to be investigated using machine learning (7,8). In the 1970s, progress with AI was temporarily halted, as the AI was only able to solve simple problems. By contrast, in the same period, expert systems consisting of knowledge bases and inference engines were invented, and tools for diagnosis in specific fields such as MYCIN and INTERNIST-1 were developed $(9,10)$. Subsequently, deep learning was proposed by Dechter (11) 
in 1986 and a convolutional neural network was proposed by LeCun et al (12) in 1988, leading to the second AI boom. In this boom, to allow adaptation to real-world problems, experts in various fields educated AI using parameters, including marketing, healthcare and life science data. In addition, surgical robots began to flourish during this period. Among them, PUMA 200 was developed to automatically identify the appropriate location of lesions in computed tomography-guided brain tumor biopsies and was the first robot used for assisting human neurosurgery (13). AESOP was a breakthrough in robotic surgery when introduced in 1994, as it was the first laparoscopic camera holder to be approved by the FDA (14). Moreover, in 2000, the da Vinci Surgical System obtained FDA approval for use in general laparoscopic procedures and became the first operative surgical robot in the US (15). In 2005, a surgical technique for the da Vinci Surgical System was documented in canine and cadaveric models called transoral robotic surgery; this was the only FDA-approved robot to perform head and neck surgery at the time (16).

In addition, medications based on a computational analysis of the crystal structure of molecules were developed $(17,18)$. The ROBODOC Surgical System was introduced and revolutionized orthopedic surgery by being able to assist with hip replacement surgeries. This was the first surgical robot to be approved for use in humans by the FDA in 2008 (19).

Thus, during the second AI boom, several tools were successfully developed. However, it was difficult for humans to provide the information that an AI needs to solve complex problems, and it was difficult for the machines of that time to learn the vast level of information available.

With the advent of deep learning in 2006 and the development of computers and communication equipment, the interest in AI was renewed $(20,21)$. In particular, the historical victory of a deep learning program by utilizing a convolutional neural network, a deep learning method in image recognition, in an image recognition contest called the ImageNet Large Scale Visual Recognition Challenge (ILSVRC) triggered the third AI boom (22). As a result, image recognition has become the most applied AI-based technology in the clinical setting. When considering clinical applications based on image recognition using AI, IB Neuro is a diagnostic software used to detect brain tumors by MRI, and this was approved by the FDA in 2008 as the first AI-based application in humans (23). In addition, IntelliSite was approved by the FDA as the first whole-slide imaging system in 2017 (24,25). Thus, a number of AI-based applications were approved by the FDA in the third AI boom. The number of approved applications for diagnostics, mainly image recognition, increased from 11 in $2008-2015$ to 135 in 2016-2020 (Table I) $(23,26)$. In addition to applications for diagnosis, applications for treatment are also being approved by the FDA, with three AI-based applications to support treatment processes such as radiotherapy being approved in 2018-2019 $(23,26)$.

Moreover, AI-based applications for follow-up of treatment progress are also being developed. In 2012, the BodyGuardian Remote Monitoring System was the first AI-based application for follow-up to be approved by the FDA (23). Subsequently, applications for follow-up are being actively developed and 13 applications were approved by the FDA in 2017-2020 $(23,26)$. Similarly, computational support continues to contribute to drug development, such as methylenetetrahydrofolate dehydrogenase 2 targeting one carbon metabolism (27).

AI is being applied to various processes in the medical field. Current AI and AI-based applications are often specialized for each field; however, applications that are widely available, such as IBM Watson (ibm.com/watson), have been also developed (28-30). These applications have been developed during each of the AI booms, each of which exhibited their own trends and problems. AI in the current boom is more developed than before, but there are also problems such as the lack of knowledge regarding the information that AI recognizes. The resolution of such problems is expected to further develop AI. Big data and AI will continue to support healthcare in numerous ways.

\section{Current AI applied in medicine}

Diagnosis. Diagnosis requires the ability to process different types of information about patients and detect abnormalities with high accuracy and reproducibility. In conventional diagnosis, physicians process various pieces of patient information using their own knowledge and/or experience, and detect abnormalities in patients using their own senses or through diagnostic equipment. This method sometimes fails to detect abnormalities in patients or results in the wrong decisions being made. In addition, the diagnostic ability is dependent on the experience of the physician. Therefore, AI is expected to have diagnostic performance with reproducibility and accuracy equal to or better than that of skilled physicians, and to compensate for differences in physician experience. Current AI for diagnosis is actively being developed to perform diagnostic imaging with computed tomography and tissue sections. In particular, convolutional neural networks perform well in the ILSVRC every year; therefore, convolutional neural networks are the most used for diagnostic imaging and perform as well as or better than skilled physicians (31). Moreover, systems have been developed to predict radiation or anticancer drug sensitivity using convolutional neural networks $(32,33)$. In addition to diagnostic imaging, AI is also being developed to diagnose diseases such as cancer via machine learning of blood components (34). However, limitations in measurement sensitivity and technical artifacts such as noise are barriers to diagnosis using blood components. The development of improved measurement technology and/or more advanced machine learning models would be required for a diagnosis that applies machine learning of blood components (35).

As aforementioned, the current application of AI for diagnosis mainly improves the accuracy of each test. By contrast, for the identification of a disease from various symptoms in a patient and the results of tests, a wide range of knowledge, not specific knowledge, and advanced information processing is necessary. To meet this demand, AI assistants such as Watson are also being developed that can learn the literature on a subject by enabling the processing of natural language, and can make complex decisions using expert systems $(28,36)$.

Thus, for diagnosis, AI is mainly developed to improve the accuracy of each test and make appropriate decisions using the large quantity of related literature available, and different algorithms suitable for each process are applied. 
A

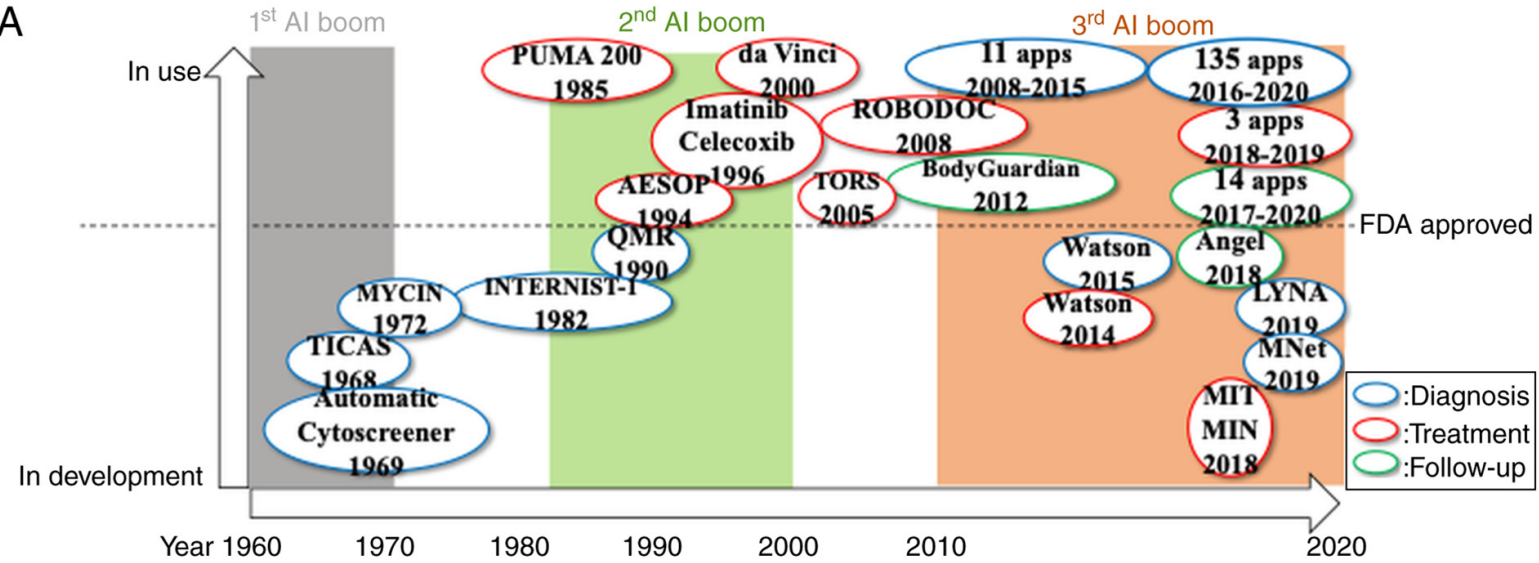

B

\begin{tabular}{|l|l|l|l|}
\hline & Trigger & Trends & Problems \\
\hline $\mathbf{1}^{\text {st }}$ AI boom & $\begin{array}{l}\text { - AI and machine } \\
\text { learning were } \\
\text { proposed (1959). }\end{array}$ & $\begin{array}{l}\text { - AI searched for conditions to achieve } \\
\text { the goal (purpose) by classifying cases } \\
\text { according to various possible routes } \\
\text { to the goal. }\end{array}$ & $\begin{array}{l}\text { - It was difficult to solve complex problems } \\
\text { (it could only solve problems determined by } \\
\text { clear rules). }\end{array}$ \\
\hline $2^{\text {nd }} \mathbf{A I}$ boom & $\begin{array}{l}\text { - Deep learning and } \\
\text { convolutional neural } \\
\text { network were } \\
\text { developed (1986, } \\
\text { 1988). }\end{array}$ & $\begin{array}{l}\text { - AI was educated by experts in various } \\
\text { fields to adapt to complex problems } \\
\text { with various parameters in the fields. }\end{array}$ & $\begin{array}{l}\text { - It required enormous costs to obtain } \\
\text { knowledge from experts in each field. } \\
\text { It was difficult even for experts to express } \\
\text { knowledge (conditions) to achieve the purpose. }\end{array}$ \\
\hline $\begin{array}{l}3^{\text {rd }} \text { AI boom } \\
\text { program was a } \\
\text { historical victory in } \\
\text { ILSVRC (2012). }\end{array}$ & $\begin{array}{l}\text { - AI extracts feature values by utilizing } \\
\text { auto-encoder without the need for } \\
\text { human knowledge representation. } \\
\text { - Abundant data is accumulated due to } \\
\text { the development of communication } \\
\text { and sensors technology (Big Data). }\end{array}$ & $\begin{array}{l}\text { - The basis for the decision of AI is difficult for } \\
\text { human to understand. } \\
\text { Machine powers with high performance are } \\
\text { necessary to handle abundant data. }\end{array}$ \\
\hline
\end{tabular}

Figure 1. (A) Historical view of clinical applications with computational support. (B) Trigger, trends and problems of each AI boom. AI, artificial intelligence; ILSVRC, ImageNet Large Scale Visual Recognition Challenge.

Treatment. For treatment, surgical robots are mainly being developed. Surgical robots are suitable for detailed work with precise movements that is beyond the reach of human hands. In conventional surgical robot algorithms, classification and detection of objects required during surgery are performed by the developer manually creating features of the region of interest; however, with the advent of deep learning, convolutional neural networks are being applied for the classification and detection of objects $(37,38)$. In addition, real-time predictions are also being made with recurrent neural networks (39). Conventional surgical robots are operated by a physician; however, surgical robots that work automatically without operation by a physician are also being developed (40). Thus, the development of AI has also led to the development of robots that support physicians during surgery.

In addition, in drug therapy, AI-based applications are being introduced in diagnosis and follow-up rather than in the treatment process. Various AI applications have been introduced in the drug development process to develop therapeutic drugs. In the drug development process, developers need to process an enormous amount of information to discover just a few promising compounds from millions to tens of millions of candidate compounds (41). Various types of AI play active roles in processing this information, as described in detail later in this review.
Thus, in the treatment process, surgical robots are mainly being developed to make the operation more accurate and reduce the burden on the physician, while in the drug development process, AI is being used to process large quantities of information.

Follow-up. In medicine, no matter what type of disease or what type of treatment is given, follow-up is more or less always necessary. In addition, life expectancy in the world has increased by 20 years in the last 50 years, and as the population ages, the risk of having chronic diseases increases. Against this background, wearable devices equipped with AI that can constantly monitor health conditions and immediately detect any abnormality in wearers are actively being developed. In particular, wearable devices are expected to be used in cardiology, where the condition of the patient may change rapidly and there is a direct link with mortality status. In fact, more than half of the applications for follow-up approved by the FDA in 2017-2020, including the ECG app on the Apple ${ }^{\mathrm{TM}}$ Watch, are wearable cardiology devices $(23,26)$. A number of the algorithms in wearable devices are applied artificial neural networks or adaptive algorithms (42).

In addition to the wearable devices, automated communication systems have also being introduced for follow-up. For example, Pharmabot was a chatbot developed in 2015 
Table I. FDA approved AI-based applications.

\begin{tabular}{|c|c|c|c|c|}
\hline Applications & Company & Purpose & Medical specialty & $\begin{array}{c}\text { FDA } \\
\text { Cleared }\end{array}$ \\
\hline IB Neuro & Imaging Biometrics, LLC & Diagnosis & Neuroradiology & 2008 \\
\hline Pathwork Tissue of Origin Test Kit-FFPE & Pathwork Diagnostics, Inc. & Diagnosis & Pathology & 2010 \\
\hline DeltaView Model 2.1 & Riverain Technologies & Diagnosis & Radiology & 2011 \\
\hline AlphaPoint Imaging Software & RadLogics, Inc. & Diagnosis & Radiology & 2012 \\
\hline BodyGuardian Remote Monitoring System & Preventice & Follow-up & Cardiology & 2012 \\
\hline ClearRead +Confirm & Riverain Technologies & Diagnosis & Radiology & 2012 \\
\hline Temporal Comparison & Riverain Technologies & Diagnosis & Radiology & 2012 \\
\hline cvi42 & $\begin{array}{l}\text { Circle Cardiovascular } \\
\text { Imaging, Inc. }\end{array}$ & Diagnosis & Radiology & 2014 \\
\hline Ahead 100 & BrainScope & Diagnosis & Neurology & 2014 \\
\hline AliveCor & AliveCor & Diagnosis & Cardiology & 2014 \\
\hline Lung Density Analysis & Imbio LLC & Diagnosis & Radiology & 2014 \\
\hline Vitrea CT Lung Density Analysis Software & Vital Images, Inc. & Diagnosis & Radiology & 2015 \\
\hline Stroke VCAR & GE Medical Systems & Diagnosis & Neuroradiology & 2016 \\
\hline QbCheck & QbTech AB & Diagnosis & Psychiatry & 2016 \\
\hline PixelShine & AlgoMedica & Diagnosis & Radiology & 2016 \\
\hline Steth IO & Stratoscientific, Inc. & Diagnosis & General medicine & 2016 \\
\hline ClearRead CT & Riverain Technologies & Diagnosis & Radiology & 2016 \\
\hline Arterys Cardio DL & Arterys Inc & Diagnosis & Radiology & 2016 \\
\hline CT CoPilot & ZepMed, LLC. & Diagnosis & Neuroradiology & 2016 \\
\hline ClearView cCAD & ClearView Diagnostics Inc. & Diagnosis & Oncology & 2016 \\
\hline Arterys Cardio DL & Arterys Inc. & Diagnosis & Radiology & 2017 \\
\hline Cantab Mobile & Cambridge Cognition, Ltd. & Diagnosis & Neurology & 2017 \\
\hline $\begin{array}{l}\text { Lung Nodule Assessment and } \\
\text { Comparison Option }\end{array}$ & Philips Medical Systems & Diagnosis & Radiology & 2017 \\
\hline EnsoSleep & EnsoData, Inc. & Diagnosis & Neurology & 2017 \\
\hline AmCAD-US & AmCad BioMed Corporation & Diagnosis & Radiology & 2017 \\
\hline QuantX & Quantitative Insights, Inc. & Diagnosis & Radiology & 2017 \\
\hline NeuroQuant & Cortechs.ai & Diagnosis & Neuroradiology & 2017 \\
\hline LesionQuant & Cortechs.ai & Diagnosis & Neuroradiology & 2017 \\
\hline Arterys Oncology DL & Arterys Inc & Diagnosis & Radiology & 2017 \\
\hline $\begin{array}{l}\text { Rooti Rx System ECG Event Recorder, } \\
\text { Rooti Link APP Software }\end{array}$ & Rooti Labs, Ltd. & Diagnosis & Cardiology & 2017 \\
\hline BioFlux & Biotricity, Inc. & Diagnosis & Cardiology & 2017 \\
\hline CNeuro cMRI & Combinostics Oy & Diagnosis & Neuroradiology & 2018 \\
\hline Idx & IDx LLC & Diagnosis & Ophthalmology & 2018 \\
\hline WAVE Clinical Platform & $\begin{array}{l}\text { Excel Medical Electronics, } \\
\text { LLC }\end{array}$ & Follow-up & $\begin{array}{l}\text { Hospital } \\
\text { monitoring }\end{array}$ & 2018 \\
\hline Insight BD & Siemens Healthineers & Diagnosis & Radiology & 2018 \\
\hline Viz LVO (ContaCT) & Viz. AI, Inc. & Diagnosis & Neuroradiology & 2018 \\
\hline DM-Density & Densitas, Inc. & Diagnosis & Oncology & 2018 \\
\hline OsteoDetect & Imagen Technologies, Inc. & Diagnosis & Radiology & 2018 \\
\hline Quantib Brain & Quantib BV & Diagnosis & Neuroradiology & 2018 \\
\hline Guardian Connect System & Medtronic & Diagnosis & Endocrinology & 2018 \\
\hline PowerLook Density Assessment Software & ICAD Inc. & Diagnosis & Radiology & 2018 \\
\hline Viz CTP & Viz. ai, inc. & Diagnosis & Neuroradiology & 2018 \\
\hline NeuralBot & Neural Analytics, Inc. & Diagnosis & Radiology & 2018 \\
\hline OsteoDetect & Imagen Technologies & Diagnosis & Radiology & 2018 \\
\hline $\begin{array}{l}\text { EchoMD Automated Ejection Fraction } \\
\text { Software }\end{array}$ & Bay Labs, Inc. & Diagnosis & Radiology & 2018 \\
\hline MindMotion GO & MindMaze SA & Follow-up & Orthopedics & 2018 \\
\hline LungQ & Thirona Corporation & Diagnosis & Radiology & 2018 \\
\hline
\end{tabular}


Table I. Continued.

\begin{tabular}{|c|c|c|c|c|}
\hline Applications & Company & Purpose & Medical specialty & $\begin{array}{c}\text { FDA } \\
\text { Cleared }\end{array}$ \\
\hline HealthCCS & Zebra Medical Vision Ltd. & Diagnosis & Radiology & 2018 \\
\hline $\begin{array}{l}\text { EchoMD Automated Ejection Fraction } \\
\text { Software }\end{array}$ & Bay Labs, Inc. & Diagnosis & Cardiology & 2018 \\
\hline DenSeeMammo & Statlife & Diagnosis & Oncology & 2018 \\
\hline DreaMed & DreaMed Diabetes, Ltd & Follow-up & Endocrinology & 2018 \\
\hline ProFound $^{\mathrm{TM}}$ AI Software V2.1 & iCAD, Inc & Diagnosis & Radiology & 2018 \\
\hline BriefCase- ICH & Aidoc Medical, Ltd. & Diagnosis & Neuroradiology & 2018 \\
\hline AmCAD-UT Detection 2.2 & AmCAD BioMed Corporation & Diagnosis & Endocrinology & 2018 \\
\hline Arterys MICA & Arterys, Inc. & Diagnosis & Radiology & 2018 \\
\hline ECG App & Apple, Inc. & Follow-up & Cardiology & 2018 \\
\hline Volpara Imaging Software & $\begin{array}{l}\text { Volpara Health Technologies } \\
\text { Limited }\end{array}$ & Diagnosis & Oncology & 2018 \\
\hline AI-ECG Platform & $\begin{array}{l}\text { Shenzhen Carewell } \\
\text { Electronics, Ltd. }\end{array}$ & Diagnosis & Cardiology & 2018 \\
\hline FibriCheck & Qompium NV & Follow-up & Cardiology & 2018 \\
\hline Irregular Rhythm Notification Feature & Apple, Inc. & Diagnosis & Cardiology & 2018 \\
\hline RightEye Vision System & RightEye, LLC & Diagnosis & Ophthalmology & 2018 \\
\hline Accipiolx & MaxQ-Al, Ltd. & Diagnosis & Radiology & 2018 \\
\hline icobrain & Icometrix NV & Diagnosis & Radiology & 2018 \\
\hline FluoroShield $^{\mathrm{TM}}$ & Omega Medical Imaging, LLC & Treatment & Radiology & 2018 \\
\hline Vitrea CT Brain Perfusion & Vital Images, Inc. & Diagnosis & Neuroradiology & 2018 \\
\hline SubtlePET & Subtle Medical, Inc. & Diagnosis & Neuroradiology & 2018 \\
\hline FerriSmart Analysis System & $\begin{array}{l}\text { Resonance Health Analysis } \\
\text { Service Pty, Ltd. }\end{array}$ & Diagnosis & Radiology & 2018 \\
\hline Embrace & Empatica Srl & Follow-up & Neurology & 2018 \\
\hline Quantib ND & Quantib BV & Diagnosis & Neuroradiology & 2018 \\
\hline iSchemaView RAPID & iSchemaView, Inc. & Diagnosis & Radiology & 2018 \\
\hline Study Watch & Verily Life Sciences LLC & Follow-up & Cardiology & 2019 \\
\hline cmTriage & CureMetrix, Inc. & Diagnosis & Oncology & 2019 \\
\hline $\begin{array}{l}\text { Thoracic VCAR with GSI Pulmonary } \\
\text { Perfusion }\end{array}$ & GE Medical Systems & Diagnosis & Radiology & 2019 \\
\hline KardiaAI & AliveCor, Inc & Follow-up & Cardiology & 2019 \\
\hline Loop System & Spry Health, Inc. & Follow-up & $\begin{array}{l}\text { Hospital } \\
\text { monitoring }\end{array}$ & 2019 \\
\hline RhythmAnalytics & Biofourmis Singapore Pte, Ltd. & Follow-up & Cardiology & 2019 \\
\hline Bone Vcar & GE Medical Systems & Diagnosis & Radiology & 2019 \\
\hline Aidoc Briefcase- ICH and PE triage & Aidoc Medical, Ltd. & Diagnosis & Radiology & 2019 \\
\hline Deep Learning Image Reconstruction & GE Medical Systems, LLC. & Diagnosis & Radiology & 2019 \\
\hline eMurmer ID & CSD Labs GmbH & Diagnosis & Cardiology & 2019 \\
\hline HealthPNX & Zebra Medical Vision Ltd. & Diagnosis & Radiology & 2019 \\
\hline Aidoc BriefCase- CSF triage & Aidoc Medical, Ltd. & Diagnosis & Radiology & 2019 \\
\hline ReSET-O & Pear Therapeutics, Inc. & Treatment & Psychiatry & 2019 \\
\hline HealthICH & Zebra Medical Vision Ltd. & Diagnosis & Neuroradiology & 2019 \\
\hline $\begin{array}{l}\text { Advanced Intelligent Clear-IQ Engine } \\
\text { (AiCE) }\end{array}$ & $\begin{array}{l}\text { Canon Medical Systems } \\
\text { Corporation }\end{array}$ & Diagnosis & Radiology & 2019 \\
\hline Koios DS & Koios Medical, Inc & Diagnosis & Oncology & 2019 \\
\hline DeepCT & Deep01 Limited & Diagnosis & Neuroradiology & 2019 \\
\hline iNtuition-Structural Heart Module & TeraRecon, Inc. & Diagnosis & Radiology & 2019 \\
\hline AI-Rad Companion (Pulmonary) & Siemens Healthineers & Diagnosis & Radiology & 2019 \\
\hline ACR I LAB Urine Analysis Test System & Healthy.io, Ltd. & Diagnosis & Urology & 2019 \\
\hline $\begin{array}{l}\text { Current Wearable Health Monitoring } \\
\text { System }\end{array}$ & Current Health, Ltd. & Follow-up & $\begin{array}{l}\text { Hospital } \\
\text { monitoring }\end{array}$ & 2019 \\
\hline
\end{tabular}


Table I. Continued.

\begin{tabular}{|c|c|c|c|c|}
\hline Applications & Company & Purpose & Medical specialty & $\begin{array}{l}\text { FDA } \\
\text { Cleared }\end{array}$ \\
\hline $\begin{array}{l}\text { physIQ Heart Rhythm and Respiratory } \\
\text { Module }\end{array}$ & physIQ, Inc & Diagnosis & Cardiology & 2019 \\
\hline RayCare 2.3 & RaySearch Laboratories AB & Treatment & Radiology & 2019 \\
\hline Critical Care Suite & GE Medical Systems & Diagnosis & Radiology & 2019 \\
\hline Biovitals Analytics Engine & Biofourmis Singapore Pte. Ltd & Follow-up & Cardiology & 2019 \\
\hline Caption Guidance & Caption Health, Inc. & Diagnosis & Radiology & 2019 \\
\hline AI-Rad Companion (cardiovascular) & Siemens Healthineers & Diagnosis & Radiology & 2019 \\
\hline SubtleMR & Subtle Medical, Inc. & Diagnosis & Radiology & 2019 \\
\hline StoneChecker & Imaging Biometrics, LLC & Diagnosis & Radiology & 2019 \\
\hline BrainScope TBI & BrainScope Company, Inc & Diagnosis & Neurology & 2019 \\
\hline ProFound AI Software V2.1 & ICAD Inc. & Diagnosis & Oncology & 2019 \\
\hline KOALA & IB Lab GmbH & Diagnosis & Radiology & 2019 \\
\hline EchoGo Core & Ultromics, Ltd. & Diagnosis & Cardiology & 2019 \\
\hline RSI-MRI+ & HealthLytix & Diagnosis & Radiology & 2019 \\
\hline HealthCXR & Zebra Medical Vision, Ltd. & Diagnosis & Radiology & 2019 \\
\hline icobrain & Icometrix NV & Diagnosis & Neuroradiology & 2019 \\
\hline QyScore Software & Qynapse & Diagnosis & Neuroradiology & 2019 \\
\hline Aidoc BriefCase- LVO & Aidoc Medical, Ltd. & Diagnosis & Neuroradiology & 2019 \\
\hline AutoMIStar & $\begin{array}{l}\text { Apollo Medical Imaging } \\
\text { Technology Pty, Ltd. }\end{array}$ & Diagnosis & Neuroradiology & 2019 \\
\hline TransparaTM & Screenpoint Medical B.V. & Diagnosis & Radiology & 2019 \\
\hline ADAS 3D & Galgo Medical S.L & Diagnosis & Radiology & 2020 \\
\hline QuantX & Quantitative Insights, Inc. & Diagnosis & Radiology & 2020 \\
\hline Eko Analysis Software & Eko Devices, Inc. & Follow-up & Cardiology & 2020 \\
\hline densitas densityai & Densitas, Inc. & Diagnosis & Radiology & 2020 \\
\hline red dot & Behold.AI Technologies, Ltd. & Diagnosis & Radiology & 2020 \\
\hline icobrain-ctp & Icometrix NV & Diagnosis & Neuroradiology & 2020 \\
\hline Broncholab & Fluidda, Inc. & Diagnosis & Radiology & 2020 \\
\hline Transpara & ScreenPoint Medical B.V. & Diagnosis & Oncology & 2020 \\
\hline Al-Rad Companion (Musculoskeletal) & Siemens Healthineers & Diagnosis & Radiology & 2020 \\
\hline Hepatic VCAR & GE Medical Systems & Diagnosis & Radiology & 2020 \\
\hline MammoScreen & Therapixel & Diagnosis & Oncology & 2020 \\
\hline RAPID ICH & iSchemaView, Inc. & Diagnosis & Neuroradiology & 2020 \\
\hline AIMI-Triage CXR PTX & RadLogics, Inc. & Diagnosis & Radiology & 2020 \\
\hline CuraRad-ICH & Keya Medical & Diagnosis & Neuroradiology & 2020 \\
\hline NinesAI & Nines, Inc. & Diagnosis & Neuroradiology & 2020 \\
\hline HealthVCF & Zebra Medical Vision, Ltd. & Diagnosis & Radiology & 2020 \\
\hline Syngo.CT CaScoring & Siemens Healthineers & Diagnosis & Radiology & 2020 \\
\hline MEDO ARIA & Medo.AI & Diagnosis & Orthopedics & 2020 \\
\hline Auto 3D Bladder Volume Tool & Butterfly Network, Inc. & Diagnosis & Urology & 2020 \\
\hline AI-Rad Companion Brain MR & Siemens Healthineers & Diagnosis & Neuroradiology & 2020 \\
\hline qER & Qure.ai Technologies & Diagnosis & Neuroradiology & 2020 \\
\hline BriefCase-IFG & Aidoc Medical, Ltd. & Diagnosis & Radiology & 2020 \\
\hline CINA & AVICENNA.AI & Diagnosis & Neuroradiology & 2020 \\
\hline Rapid ASPECTS & iSchemaView Inc. & Diagnosis & Neuroradiology & 2020 \\
\hline EyeArt & Eyenuk, Inc. & Diagnosis & Ophthalmology & 2020 \\
\hline InferRead Lung CT.AI & $\begin{array}{l}\text { Beijing Infervision Technology } \\
\text { Co., Ltd. }\end{array}$ & Diagnosis & Radiology & 2020 \\
\hline Rapid LVO 1.0 & iSchemaView, Inc. & Diagnosis & Neuroradiology & 2020 \\
\hline HealthMammo & Zebra Medical Vision, Ltd. & Diagnosis & Oncology & 2020 \\
\hline $\begin{array}{l}\text { Caption Interpretation Automated Ejection } \\
\text { Fraction Software }\end{array}$ & Caption Health & Diagnosis & Cardiology & 2020 \\
\hline
\end{tabular}


Table I. Continued.

\begin{tabular}{|c|c|c|c|c|}
\hline Applications & Company & Purpose & Medical specialty & $\begin{array}{c}\text { FDA } \\
\text { Cleared }\end{array}$ \\
\hline AI-Rad Companion Prostate MR & Siemens Healthineers & Diagnosis & Radiology & 2020 \\
\hline FractureDetect (FX) & Imagen Technologies & Diagnosis & Radiology & 2020 \\
\hline VIDAlvision & VIDA Diagnostics, Inc. & Diagnosis & Radiology & 2020 \\
\hline Accipiolx & MaxQ Al, Ltd. & Diagnosis & Neuroradiology & 2020 \\
\hline Aidoc BriefCase for iPE Triage & Aidoc Medical, Ltd. & Diagnosis & Radiology & 2020 \\
\hline Aview 2.0 & Coreline Soft Co., Ltd. & Diagnosis & Radiology & 2020 \\
\hline AVA (Augmented Vascular Analysis) & $\begin{array}{l}\text { See-Mode Technologies } \\
\text { Pte, Ltd. }\end{array}$ & Diagnosis & Cardiology & 2020 \\
\hline THINQ & CorticoMetrics LLC & Diagnosis & Neuroradiology & 2020 \\
\hline Cleerly Labs V2.0 & Cleerly, Inc. & Diagnosis & Radiology & 2020 \\
\hline Syngo.CT Neuro Perfusion & Siemens Healthineers & Diagnosis & Neuroradiology & 2020 \\
\hline Quantib Prostate & Quantib BV & Diagnosis & Radiology & 2020 \\
\hline AVIEW LCS & Coreline Soft Co., Ltd. & Diagnosis & Radiology & 2020 \\
\hline Liver Surface Nodularity (LSN) & Imaging Biometrics, LLC & Diagnosis & Radiology & 2020 \\
\hline WRDensity & Whiterabbit.ai Inc. & Diagnosis & Oncology & 2020 \\
\hline Neuro.AI Algorithm & TeraRecon, Inc. & Diagnosis & Neuroradiology & 2020 \\
\hline FastStroke, CT Perfusion 4D & GE Medical Systems & Diagnosis & Neuroradiology & 2020 \\
\hline PROView & GE Medical Systems & Diagnosis & Radiology & 2020 \\
\hline Genius AI Detection & Hologic, Inc. & Diagnosis & Radiology & 2020 \\
\hline HALO & NiCo-Lab B.V. & Diagnosis & Neuroradiology & 2020 \\
\hline HealthJOINT & Zebra Medical Vision, Ltd. & Diagnosis & Radiology & 2020 \\
\hline HepaFat-AI & $\begin{array}{l}\text { Resonance Health Analysis } \\
\text { Service Pty, Ltd. }\end{array}$ & Diagnosis & Radiology & 2020 \\
\hline SQuEEZ Software & Cardiowise, Inc. & Diagnosis & Radiology & 2020 \\
\hline EchoGo Pro & Ultromics, Ltd. & Diagnosis & Cardiology & 2020 \\
\hline AI Metrics & AI Metrics, LLC & Diagnosis & Radiology & 2020 \\
\hline BrainInsight & Hyperfine Research, Inc. & Diagnosis & Neuroradiology & 2021 \\
\hline HearFlow Analysis & HeartFlow, Inc. & Diagnosis & Radiology & 2021 \\
\hline uAI EasyTriage-Rib & $\begin{array}{l}\text { Shanghai United Imaging } \\
\text { Intelligence Co., Ltd. }\end{array}$ & Diagnosis & Radiology & 2021 \\
\hline Visage Breast Density & Visage Imaging GmbH & Diagnosis & Oncology & 2021 \\
\hline CLEWICU System & CLEW Medical, Ltd. & Diagnosis & Hematology & 2021 \\
\hline qp-Prostate & Quibim & Diagnosis & Radiology & 2021 \\
\hline Lvivo Software Application & DiA Imaging Analysis, Ltd. & Diagnosis & Cardiology & 2021 \\
\hline Veolity & MeVis Medical Solutions AG & Diagnosis & Radiology & 2021 \\
\hline NinesMeasure & Nines, Inc. & Diagnosis & Radiology & 2021 \\
\hline $\begin{array}{l}\text { Optellum Virtual Nodule Clinic, Optellum } \\
\text { Software, Optellum Platform }\end{array}$ & Optellum, Ltd. & Diagnosis & Radiology & 2021 \\
\hline Imbio RV/LV Software & Imbio LLC & Diagnosis & Radiology & 2021 \\
\hline Vbrain & Vysioneer, Inc. & Diagnosis & Neuroradiology & 2021 \\
\hline Viz ICH & Viz. AI, Inc. & Diagnosis & Neuroradiology & 2021 \\
\hline syngo.CT Lung CAD (VD20) & Subtle Medical, Inc. & Diagnosis & Radiology & 2021 \\
\hline Saige-Q & DeepHealth & Diagnosis & Oncology & 2021 \\
\hline MEDO- Thyroid & Medo.AI & Diagnosis & Endocrinology & 2021 \\
\hline CINA CHEST & AVICENNA.AI & Diagnosis & Radiology & 2021 \\
\hline Overjet Dental Assist & Overjet, Inc. & Diagnosis & Radiology & 2021 \\
\hline
\end{tabular}

$\mathrm{AI}$, artificial intelligence.

to assist in medication education for pediatric patients and their parents using a Left-Right parsing algorithm and Care
Angel, which applied an automated voice dialogue system to check on the condition of the person requiring care, to 
Table II. Comparison between conventional and computational medicine.

\begin{tabular}{|c|c|c|}
\hline Medicine & Conventional & Computational \\
\hline Diagnosis & $\begin{array}{l}\text { Doctors meet patients; patients are diagnosed } \\
\text { using diagnostic equipment at the hospital; } \\
\text { diagnostic accuracy depends on the } \\
\text { experience of a doctor }\end{array}$ & $\begin{array}{l}\text { AI can detect lesions with the same or better accuracy than a } \\
\text { skilled doctor; AI support is used to avoid misdiagnoses; } \\
\text { AI supports the decision of the doctor by processing of the } \\
\text { medical literature }\end{array}$ \\
\hline Treatment & $\begin{array}{l}\text { Doctors perform surgery directly; doctors or } \\
\text { nurses administer medications }\end{array}$ & $\begin{array}{l}\text { Robotics support doctors in surgery; devices automatically } \\
\text { administer medications based on time and symptoms }\end{array}$ \\
\hline Follow-up & $\begin{array}{l}\text { Doctors or nurses meet patients; patients are } \\
\text { diagnosed using diagnostic equipment at a } \\
\text { hospital }\end{array}$ & $\begin{array}{l}\text { Devices, such as those that are wearable, can detect } \\
\text { abnormalities at a very early stage; the AI can consult with } \\
\text { patients about their medical conditions and medications }\end{array}$ \\
\hline Advantages & $\begin{array}{l}\text { Patients can meet their doctors and nurses; } \\
\text { abstract expression is possible }\end{array}$ & $\begin{array}{l}\text { AI decreases the burden on doctors and nurses; AI responds } \\
\text { quickly in an emergency }\end{array}$ \\
\hline Problems & $\begin{array}{l}\text { The burden on doctors and nurses is heavy; } \\
\text { sometimes it is not possible to respond } \\
\text { immediately in an emergency }\end{array}$ & $\begin{array}{l}\text { The process of outputting the data is incomprehensible } \\
\text { to humans. } \\
\text { Application cost is high. } \\
\text { AI cannot take responsibility for mistakes. }\end{array}$ \\
\hline
\end{tabular}

AI, artificial intelligence.

detect abnormalities and to alert the caregiver to changes if necessary $(43,44)$.

As aforementioned, AI-based applications are contributing to medicine in numerous ways. The characteristics of conventional medicine and AI-based medicine are summarized in Table II. In the current section, some examples of the applied AI-based applications have been introduced; however, it is difficult for AI-based medicine to classify diseases and symptoms that are still difficult to define, and to obtain the sense of security that is felt upon meeting a doctor or nurse directly. It would be possible to realize better medical care by solving the disadvantages of both and integrating the advantages of both.

\section{AI in drug development as a foundation for drug therapy}

Medications used in pharmacotherapy are discovered from among millions to tens of millions of candidate compounds after long years of research and after a huge amount of money has been expended. To evaluate each candidate compound, a number of key pieces of information are required for evaluation, such as in vitro and in vivo pharmacological activity, safety, target specificity, pharmacokinetics, physical properties such as molecular weight and solubility, stability in the body and during storage, and development cost. Therefore, drug development sites always generate enormous amounts of information. Developers need to extract the important information from this in order to discover optimal compounds. Therefore, it is expected that AI would improve the accuracy and efficiency of developers in drug development. AI was introduced to the field of drug development earlier than to the medical field (45). In conventional drug development, developers propose hypotheses for the treatment of a disease and focus on a target that can be used to develop a therapeutic drug within the hypothesis; this is followed by drug development. However, this process might miss crucial therapeutic targets and drugs for various diseases. In AI-based drug development, AI can propose, and lead to the development of, important targets and candidate drugs for disease therapy (Table III). In particular, Watson is able to identify connections and relationships among diseases, drugs, genes and other factors, and can generate novel hypotheses by mining the scientific literature (28). This tool is useful not only for drug development, but also for drug repurposing. In addition, Watson is constantly and automatically updated. Automatic learning in AI is important, not only to decrease the effort involved, but also to create better methods to meet unmet needs in life sciences and medicine.

AI-based drug development can save time and money. In conventional drug development, screening is performed using millions to tens of millions of compound libraries, followed by synthetic development based on the candidate compounds obtained from the screening and the re-evaluation of their activities to identify those with promise. However, numerous identified compounds do not exhibit physical properties and safety profiles that are suitable for pharmaceutical applications; therefore, other compounds are often re-synthesized. To avoid such time and money loss, AI-based drug development can predict the activity, physical properties and safety of each compound using computers. Various AI-based applications have been developed to predict these parameters (Figs. 2 and 3) (28,46-62). Furthermore, applications have been developed that predict not only the properties of individual compounds, but also suitable routes of synthesizing pharmacological reagents or therapeutic compounds $(61,62)$. The cost of drug development has been decreased by these applications; however, the accuracy of the AI prediction of the compound properties is not sufficient and further improvement of this factor is necessary. In particular, AI-based applications have been actively developed as screening steps, which require time and money. However, it is difficult to predict the affinities 
Table III. Comparison between conventional and AI-based drug development.

\begin{tabular}{lll}
\hline $\begin{array}{l}\text { Drug development } \\
\text { Driving factor }\end{array}$ & \multicolumn{1}{c}{ Conventional } & \multicolumn{1}{c}{ AI-based } \\
Targets & $\begin{array}{l}\text { Target-driven } \\
\text { Easily druggable targets with known structure } \\
\text { and interactions in cells }\end{array}$ & $\begin{array}{l}\text { Data-driven } \\
\text { Meaningful targets extracted by machine learning } \\
\text { using big data } \\
\text { Advantages }\end{array}$ \\
$\begin{array}{l}\text { It is easy for humans to understand identified } \\
\text { targets and compounds. }\end{array}$ & $\begin{array}{l}\text { Saves time and money by predicting the activities and } \\
\text { properties of compounds before synthesis; compounds } \\
\text { that target un-druggable molecules may be identified }\end{array}$ \\
& $\begin{array}{l}\text { Targets are limited by the complex and/or } \\
\text { un-known structures and interactions; the }\end{array}$ & $\begin{array}{l}\text { A large amount of accurate data is necessary for } \\
\text { learning; AI cannot understand whether compounds } \\
\text { aro meaningful for humans }\end{array}$ \\
& $\begin{array}{l}\text { identification of promising compounds is time } \\
\text { consuming (numerous synthesis-evaluation }\end{array}$ & \\
& cycles). &
\end{tabular}

AI, artificial intelligence.

between a target protein and compounds, for the following reasons: i) Difficulties in predicting protein flexibility; ii) ambiguity regarding the complexity of a protein in an actual environment, and iii) difficulties in assessing the solvent effects of an actual environment $(63,64)$. To solve these problems, a number of experiments and new algorithms would be necessary.

One of the most difficult steps in the process of drug development is the prediction of adverse effects. It has been reported that computational modeling using machine learning is useful for predicting adverse effects (65). Moreover, it is possible to manufacture synthetic patients and data artificially by analyzing existing data using machine learning techniques $(66,67)$. As there are no ethical concerns regarding the privacy and costs of using synthetic data, this would be a powerful tool for clinical studies that require a large number of patients and may be an effective alternative for preparing training data for machine learning algorithms. These fields of research could be further enriched by AI in the near future and would also contribute to the realization of personalized precision medicine.

\section{Perspectives for AI-based medicine and drug development}

AI has been used for clinical purposes and drug development. However, the current AI-based applications are only being developed for specific applications at each stage of pharmacological or medical applications. In particular, AI-based applications with a high accuracy for diagnosis have actively been developed. However, a diagnosis is not determined based only on the result of one diagnostic method, and should be performed by comprehensively combining various types of information, such as chief complaints and physical findings; a system that integrates the various specific diagnostic data would be necessary in the future (Fig. 3A). If the disease remained unclear based only on the acquired information, the system would be a present a diagnostic method to determine the condition. The system would avoid missing information and improve the accuracy of the diagnosis. In addition, the system would not only be used for diagnosis, but also for monitoring the progress of treatment after surgery and drug therapy. At present, almost no AI-based application has been developed that predicts the therapeutic effect or proposes a therapeutic method. However, a system has been reported in basic research that predicts the sensitivity of anticancer agents and radiation therapy based on phase contrast image information $(32,33)$. In the near future, AI may be able to predict the therapeutic effects of various therapeutic methods in advance and suggest an appropriate therapeutic method. Thus, clinical AI in the future should be a system that interprets and integrates various types of clinical information and considers the changes caused by each treatment, to promote the best flow toward the complete cure of the disease. In addition, by constantly collecting information on complaints and physical status, both inside and outside the hospital, AI systems should always support the ability of a patient to live without aggravating their condition.

In drug development, numerous specific AI-based applications are already in use. In particular, a number of applications for the simulation of the docking of compounds to target proteins have been developed (47-53). Moreover, in addition to having an excellent specific score (such as affinity for a target protein), a candidate compound needs to have comprehensive excellent safety and pharmacokinetics. Therefore, a system for identifying promising compounds by considering various characteristics, as well as learning specific scores, will be required in the future (Fig. 3B). Furthermore, in drug development, clinical trials impose a heavy financial and time burden. It is necessary to improve the efficiency of clinical trials by using portal devices, which configure and manage devices remotely over the network or via USB connection, and collecting and selecting applicable patients.

The most important key to solving the problems facing AI-based applications is making it possible for people to understand the judgment process of AI. AI-based clinical applications would be utilized in important aspects of future treatment decisions, such as the diagnosis and evaluation of treatment effectiveness. If the AI determination process is 


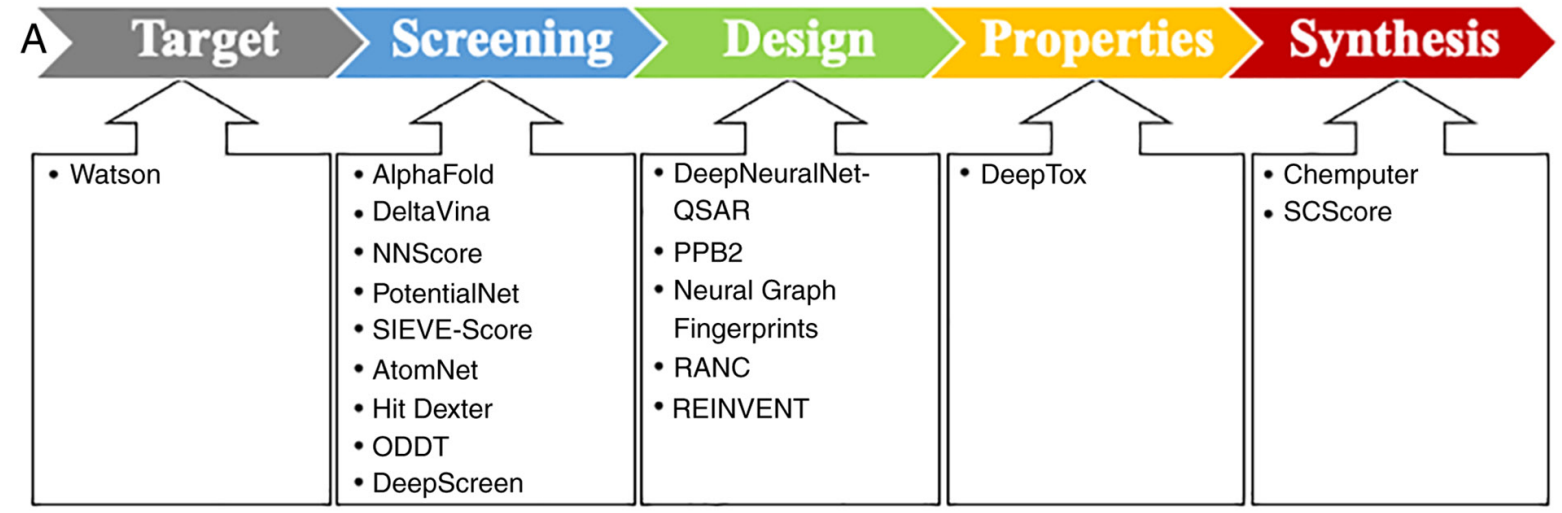

B

\begin{tabular}{|c|c|c|c|}
\hline Process & Al-based Tool & Description & References \\
\hline Target & Watson & $\begin{array}{l}\text { Automated hypothesis generation based on mining } \\
\text { scientific literatures. }\end{array}$ & (28) \\
\hline \multirow{9}{*}{ Screening } & AlphaFold & Protein 3D structure prediction & (46) \\
\hline & DeltaVina & $\begin{array}{l}\text { A scoring function for rescoring protein-ligand } \\
\text { binding affinity }\end{array}$ & (47) \\
\hline & NNScore & $\begin{array}{l}\text { Neural network-based scoring function for protein- } \\
\text { ligand interactions }\end{array}$ & (48) \\
\hline & PotentialNet & $\begin{array}{l}\text { Ligand-binding affinity prediction based on a graph } \\
\text { convolutional neural network (CNN) }\end{array}$ & (49) \\
\hline & SIEVE-Score & $\begin{array}{l}\text { An improved method of structure-based virtual } \\
\text { screening via interaction-energy-based learning }\end{array}$ & (50) \\
\hline & AtomNet & $\begin{array}{l}\text { A technology that can predict billions of small } \\
\text { molecule-protein interactions }\end{array}$ & (51) \\
\hline & Hit Dexter & $\begin{array}{l}\text { ML models for the prediction of molecules which might } \\
\text { respond to biochemical assays }\end{array}$ & (52) \\
\hline & ODDT & $\begin{array}{l}\text { A comprehensive toolkit for use in chemo-informatics } \\
\text { and molecular modeling }\end{array}$ & (53) \\
\hline & DeepScreen & $\begin{array}{l}\text { A system for predicting pharmacological activities of } \\
\text { compounds from their } 2 \mathrm{D} \text { structures }\end{array}$ & (54) \\
\hline \multirow{5}{*}{ Design } & DeepNeuralNet- QSAR & Molecular activity predictions & (55) \\
\hline & PPB2 & Poly-pharmacology prediction & (56) \\
\hline & Neural Graph Fingerprints & Property prediction of novel molecules & (57) \\
\hline & RANC & $\begin{array}{l}\text { An efficient molecular generation tool to create } \\
\text { molecules with desired properties }\end{array}$ & (58) \\
\hline & REINVENT & $\begin{array}{l}\text { Molecular de novo design using RNN (recurrent neural } \\
\text { network) and RL (reinforcement learning) }\end{array}$ & (59) \\
\hline Properties & DeepTox & Toxicity predictions & (60) \\
\hline \multirow{2}{*}{ Synthesis } & Chemputer & $\begin{array}{l}\text { A more standardized format for reporting a chemical } \\
\text { synthesis procedure }\end{array}$ & (61) \\
\hline & SCScore & $\begin{array}{l}\text { A scoring function to evaluate the synthesis complexity } \\
\text { of a molecule }\end{array}$ & (62) \\
\hline
\end{tabular}

Figure 2. (A) AI-based tools in the drug development flow. (B) Description of AI-based tools in each process. AI, artificial intelligence.

unclear, the medical staff could not evaluate the validity of the AI determination, which would lead to the distrust of AI. To solve the uncertainty of AI, 'Explainable AI' has been actively researched (68). The development of Explainable AI would be essential for the widespread use of AI-based applications in healthcare. 
A

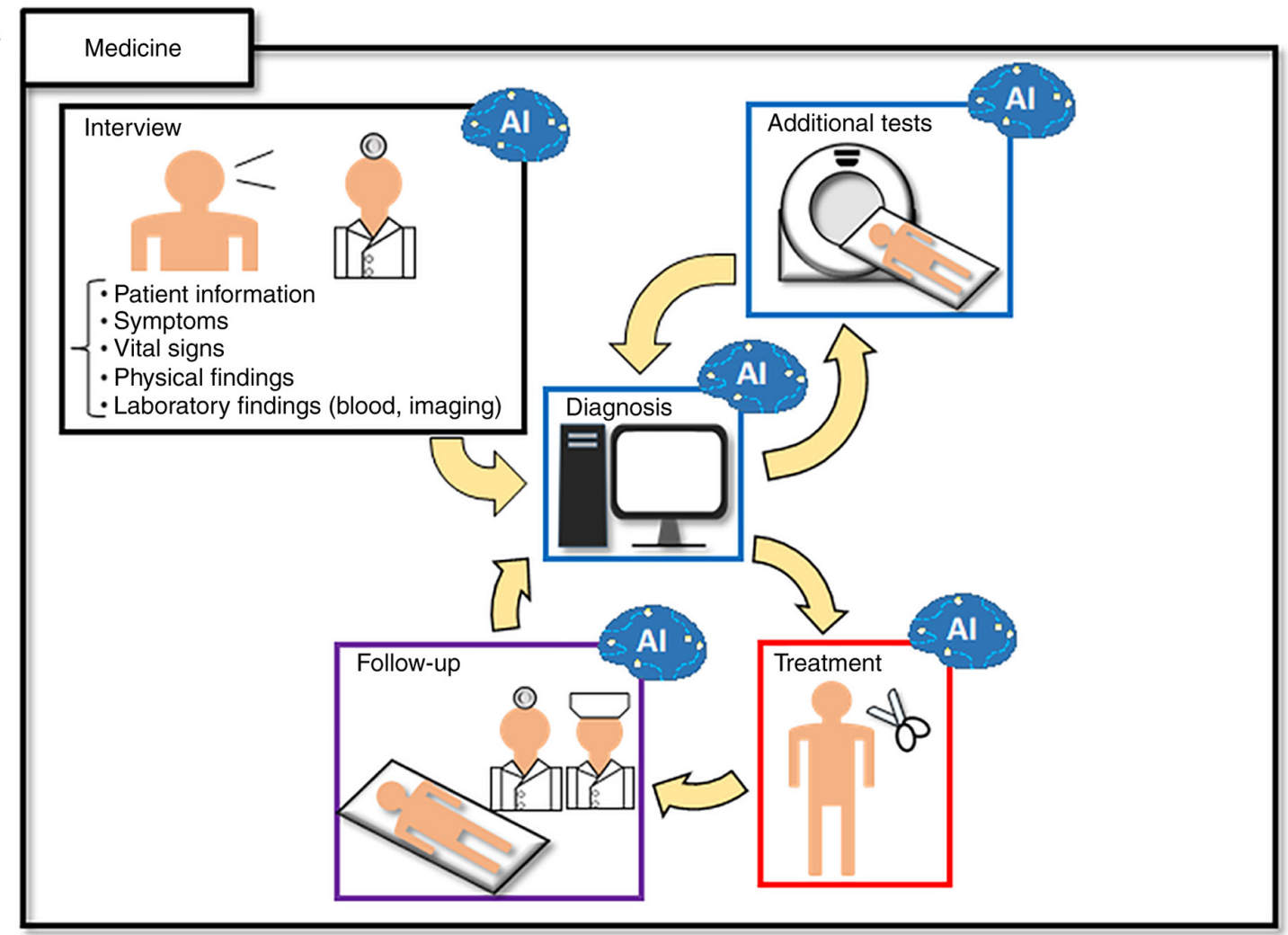

B

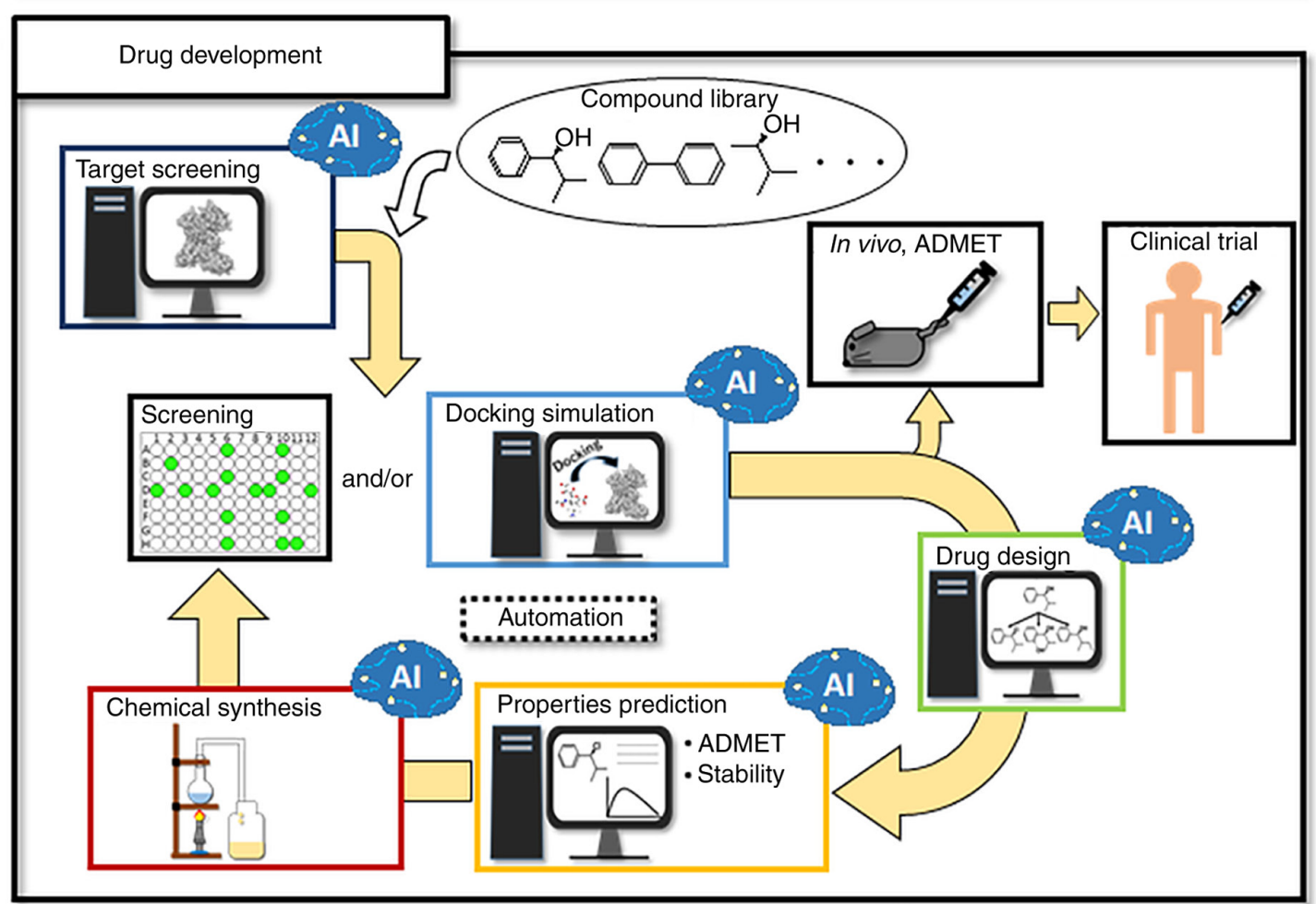

: Steps supported by Al

Figure 3. Perspectives for AI-based (A) medicine and (B) drug development. AI, artificial intelligence. ADMET, Absorption Distribution Metabolism Excretion Toxicity.

In addition, as AI-based healthcare would accumulate a greater amount of medical information than the current healthcare system, it would be necessary to prepare an infrastructure and security systems to handle large amounts of information. Futuristic clinical AI might monitor not only medical information, but also the tasks of patients and medical staff, and might forecast workflow bottlenecks.

As aforementioned, the implementation of AI could facilitate more accuracy and greater efficiency in various fields of healthcare; however, it has some issues and limitations. Both 
medical staff and developers would need to understand the issues and limitations, and then the coexistence of humans and AI could lead to better healthcare.

\section{Conclusion}

AI has evolved with the times and has been utilized in applications in drug development and healthcare. These applications are steadily producing results, and the use of AI is becoming established. The implementation of AI in society will need to overcome issues such as how to develop leading companies and train data scientists. However, company development may still face some obstacles, such as implementing AI, employment and cost. In the future, to improve human health, we should not only develop AI, but also think about the coexistence of humans with AI.

\section{Acknowledgements}

Not applicable.

\section{Funding}

This study was supported in part by a Grant-in-Aid for Scientific Research from the Ministry of Education, Culture, Sports, Science and Technology (grant nos. 19K22658, 19K09172, 19K07688, 20H00541, $221 \mathrm{~S} 0002$ and 21K19526) and the Japan Agency for Medical Research and Development (grant no. 16cm0106414h0001). Partial support was received from the Princess Takamatsu Cancer Research Fund (2019).

\section{Availability of data and materials}

Not applicable.

\section{Authors' contributions}

HI, MT and AV conceptualized the study. AA, MK and HI wrote the manuscript. All authors have read and approved the final version of the manuscript. Data authentication is not applicable.

\section{Ethics approval and consent to participate}

Not applicable.

\section{Patient consent for publication}

Not applicable.

\section{Competing interests}

Institutional endowments were received partially from Hirotsu Bio Science Inc. (Tokyo, Japan), Kinshu-kai Medical Corporation (Osaka, Japan), IDEA Consultants, Inc. (Tokyo, Japan), Kyowa-kai Medical Corporation (Hyogo, Japan), Unitech Co., Ltd. (Chiba, Japan), Chugai Co., Ltd. (Tokyo, Japan), Yakult Honsha Co., Ltd. (Tokyo, Japan) and Ono Pharmaceutical Co., Ltd. (Osaka, Japan).

\section{References}

1. Santos DP and Baeßler B: Big data, artificial intelligence, and structured reporting. Eur Radiol Exp 2: 42, 2018.

2. Akyazi T, Goti A, Oyarbide A, Alberdi E and Bayon F: A guide for the food industry to meet the future skills requirements emerging with industry 4.0. Foods 9: 492, 2020.

3. Favaretto M, Shaw D, De Clercq E, Joda T and Elger BS: Big data and digitalization in dentistry: A systematic review of the ethical issues. Int J Environ Res Public Health 17: 2495, 2020.

4. Etienne H, Hamdi S, Le Roux M, Camuset J, Khalife-Hocquemiller T, Giol M, Debrosse D and Assouad J: Artificial intelligence in thoracic surgery: Past, present, perspective and limits. Eur Respir Rev 29: 200010, 2020.

5. McCarthy J, Minsky ML, Rochester N and Shannon CE: A Proposal for the Dartmouth Summer Research Project on Artificial Intelligence, August 31, 1955. AI Magazine 27: 12, 2006.

6. Samuel AL: Some studies in machine learning using the game of checkers. IBM J Res Dev 3: 210-229, 1959.

7. WiedGL,BahrGF,OldfieldDG andBartels PH:Computer-assisted identification of cells from uterine adenocarcinoma. A clinical feasibility study with TICAS. I. Measurements at wavelength $530 \mathrm{~nm}$. Acta Cytol 12: 357-370, 1968.

8. Ishiyama T, Tsubura E, Hirao F, Yamamura Y, Takeda S, Tateisi K, Yamamoto M, Uemura M, Yaida K, Hayakawa F, et al: A study of the automation of cytodiagnosis. Med Biol Eng 7: 297-306, 1969.

9. Shortliffe EH, Axline SG, Buchanan BG, Merigan TC and Cohen SN: An artificial intelligence program to advise physicians regarding antimicrobial therapy. Comput Biomed Res 6: 544-560, 1973.

10. Miller RA, Pople HE and Myers JD: INTERNIST-1, An experimental computer-based diagnostic consultant for general internal medicine. N Engl J Med 307: 478-486, 1982.

11. Dechter R: Learning while searching in constraint-satisfaction-problems. In: Proceedings of the Fifth National Conference on Artificial Intelligence. American Association for Artificial Intelligence, Philadelphia, pp178-183, 1986.

12. LeCun Y, Boser B, Denker JS, Henderson D, Howard RE, Hubbard W and Jackel LD: Backpropagation applied to handwritten zip code recognition. Neural Comput 1: 541-551, 1989.

13. Kwoh YS, Hou J, Jonckheere EA and Hayati S: A robot with improved absolute positioning accuracy for CT guided stereotactic brain surgery. IEEE Trans Biomed Eng 35: 153-160, 1988.

14. Jacobs LK, Shayani V and Sackier JM: Determination of the learning curve of the AESOP robot. Surg Endosc 11: 54-55, 1997.

15. Sung GT and Gill IS: Robotic laparoscopic surgery: A comparison of the DA Vinci and Zeus systems. Urology 58: 893-898, 2001.

16. Weinstein GS, O'malley BW Jr and Hockstein NG: Transoral robotic surgery: Supraglottic laryngectomy in a canine model. Laryngoscope 115: 1315-1319, 2005.

17. Kurumbail RG, Stevens AM, Gierse JK, McDonald JJ, Stegeman RA, Pak JY, Gildehaus D, Miyashiro JM, Penning TD, Seibert K, et al: Structural basis for selective inhibition of cyclooxygenase-2 by anti-inflammatory agents. Nature 384: 644-648, 1996.

18. Molnár L and Losonczy H: Tyrosine kinase inhibitor STI571: New possibility in the treatment of chronic myeloid leukemia. Orv Hetil 143: 2379-2384, 2002 (In Hu).

19. Lanfranco AR, Castellanos AE, Desai JP and Meyers WC: Robotic surgery: A current perspective. Ann Surg 239: 14-21, 2004.

20. Hinton GE, Osindero S and The YW: A fast learning algorithm for deep belief nets. Neural Comput 18: 1527-1554, 2006.

21. Hinton GE and Salakhutdinov RR: Reducing the dimensionality of data with neural networks. Science 313: 504-507, 2006.

22. Komura D and Ishikawa S: Machine learning approaches for pathologic diagnosis. Virchows Arch 475: 131-138, 2019.

23. American College of Radiology. Cleared AI Algorithms. 2021. https://models.acrdsi.org. Accessed July 1, 2021.

24. U.S. Food and Drug Administration: FDA allows marketing of first whole slide imaging system for digital pathology. 2017. ttps:// www.fda.gov/news-events/press-announcements/fda-allowsmarketing-first-whole-slide-imaging-system-digital-pathology. Accessed July 1,2021.

25. Mills AM, Gradecki SE, Horton BJ, Blackwell R, Moskaluk CA, Mandell JW, Mills SE and Cathro HP: Diagnostic efficiency in digital pathology: A comparison of optical versus digital assessment in 510 surgical pathology cases. Am J Surg Pathol 42: 53-59, 2018. 
26. The Medical Futurist: FDA-approved A.I.-based algorithms 2021. https://medicalfuturist.com/fda-approved-ai-based-algorithms/. Accessed July 1,2021.

27. Asai A, Koseki J, Konno M, Nishimura T, Gotoh N, Satoh T, Doki Y, Mori M and Ishii H: Drug discovery of anticancer drugs targeting methylenetetrahydrofolate dehydrogenase 2 . Heliyon 4 e01021, 2018

28. Spangler S, Wilkins AD, Bachman BJ, Nagarajan M, Dayaram T, Haas P, Regenbogen S, Pickering CR, Comer A, Myers JN et al: Automated hypothesis generation based on mining scientific literature. KDD'14: Proceedings of the 20th ACM SIGKDD international conference on Knowledge discovery and data mining: 1877-1886, 2014.

29. Doyle-Lindrud S: Watson will see you now: A supercomputer to help clinicians make informed treatment decisions. Clin J Oncol Nurs 19: 31-32, 2015.

30. Tojo A: Clinical sequencing in leukemia with the assistance of artificial intelligence. Rinsho Ketsueki 58: 1913-1917, 2017 (In Japanese).

31. Esteva A, Kuprel B, Novoa RA, Ko J, Swetter SM, Blau HM and Thrun S: Dermatologist-level classification of skin cancer with deep neural networks. Nature 542: 115-118, 2017.

32. Toratani M, Konno M, Asai A, Koseki J, Kawamoto K, Tamari K, Li Z, Sakai D, Kudo T, Satoh T, et al: A convolutional neural network uses microscopic images to differentiate between mouse and human cell lines and their radioresistant clones. Cancer Res 78: 6703-6707, 2018

33. Yanagisawa $K$, Toratani $M$, Asai A, Konno M, Niioka H, Mizushima T, Satoh T, Miyake J, Ogawa K, Vecchione A, et al: Convolutional neural network can recognize drug resistance of single cancer cells. Int J Mol Sci 21: 3166, 2020.

34. Tanos R, Tosato G, Otandault A, Al Amir Dache Z, Pique Lasorsa L, Tousch G, El Messaoudi S, Meddeb R, Assaf MD, Ychou M, et al: Machine learning-assisted evaluation of circulating DNA quantitative analysis for cancer screening. Adv Sci (Weinh) 7: 2000486, 2020

35. Im YR, Tsui DWY, Diaz LA Jr and Wan JCM: Next-generation liquid biopsies: Embracing data science in oncology. Trends Cancer 7: 283-292, 2021

36. Harada Y and Shimizu T: Impact of a Commercial Artificial Intelligence-Driven Patient Self-Assessment Solution on Waiting Times at General Internal Medicine Outpatient Departments: Retrospective Study. JMIR Med Inform 8: e21056, 2020.

37. Litjens G, Kooi T, Bejnordi BE, Setio AAA, Ciompi F, Ghafoorian M, van Der Laak JA, van Ginneken B and Sánchez CI: A survey on deep learning in medical image analysis. Med Image Anal 42: 60-88, 2017

38. Khosravi P, Kazemi E, Imielinski M, Elemento $\mathrm{O}$ and Hajirasouliha I: Deep convolutional neural networks enable discrimination of heterogeneous digital pathology images. EBioMedicine 27: 317-328, 2018.

39. Meyer A, Zverinski D, Pfahringer B, Kempfert J, Kuehne T, Sündermann SH, Stamm C, Hofmann T, Falk V and Eickhoff C: Machine learning for real-time prediction of complications in critical care: A retrospective study. Lancet Respir Med 6: 905-914, 2018.

40. Saeidi H, Opfermann JD, Kam M, Raghunathan S, Leonard S and Krieger A: A confidence-based shared control strategy for the smart tissue autonomous robot (STAR). Rep US 2018: 1268-1275, 2018.

41. Brown N, Cambruzzi J, Cox PJ, Davies M, Dunbar J, Plumbley D, Sellwood MA, Sim A, Williams-Jones BI, Zwierzyna M and Sheppard DW: Big data in drug discovery. Prog Med Chem 57: 277-356, 2018

42. Vélez-Guerrero MA, Callejas-Cuervo M and Mazzoleni S Artificial intelligence-based wearable robotic exoskeletons for upper limb rehabilitation: A review. Sensors (Basel) 21: 2146, 2021.

43. Comendador B, Francisco B, Medenilla J, Nacion S and Serac T: Pharmabot: A pediatric generic medicine consultant chatbot. J Automat Control Eng 3: 137-140, 2015.

44. Wong V, Rosenbaum S, Sung W, Kaplan RM, Bott N, Platchek T, Milstein A and Shah NR: Caring for caregivers: bridging the gap between family caregiving policy and practice. NEJM Catal Innov Care Deliv 2: 2021.

45. Paul D, Sanap G, Shenoy S, Kalyane D, Kalia K and Tekade RK: Artificial intelligence in drug discovery and development. Drug Discov Today 26: 80-93, 2021

46. AlQuraishi M: AlphaFold at CASP13. Bioinformatics 35 4862-4865, 2019.
47. Wang $\mathrm{C}$ and Zhang Y: Improving scoring-docking-screening powers of protein-ligand scoring functions using random forest. J Comput Chem 38: 169-177, 2017.

48. Durrant JD and McCammon JA: NNScore 2.0: A neural-network receptor-ligand scoring function. J Chem Inf Model 51: 2897-2903, 2011.

49. Feinberg EN, Sur D, Wu Z, Husic BE, Mai H, Li Y, Sun S, Yang J, Ramsundar B and Pande VS: PotentialNet for molecular property prediction. ACS Cent Sci 4: 1520-1530, 2018.

50. Yasuo N and Sekijima M: Improved method of structure-based virtual screening via interaction-energy-based learning. J Chem Inf Model 59: 1050-1061, 2019.

51. Wallach I, Dzamba M and Heifets A: AtomNet: A deep convolutional neural network for bioactivity prediction in structure-based drug discovery. arXiv: $1510.02855,2015$.

52. Stork C, Chen Y, Sícho M and Kirchmair J: Hit dexter 2.0 Machine-learning models for the prediction of frequent hitters. J Chem Inf Model 59: 1030-1043, 2019.

53. Wójcikowski M, Zielenkiewicz P and Siedlecki P: Open drug discovery toolkit (ODDT): A new open-source player in the drug discovery field. J Cheminform 7: 26, 2015.

54. Rifaioglu AS, Nalbat E, Atalay V, Martin MJ, Cetin-Atalay R and Doğan T: DEEPScreen: High performance drug-target interaction prediction with convolutional neural networks using 2-D structural compound representations. Chem Sci 11: 2531-2557, 2020.

55. Xu Y, Ma J, Liaw A, Sheridan RP and Svetnik V: Demystifying multitask deep neural networks for quantitative structure-activity relationships. J Chem Inf Model 57: 2490-2504, 2017.

56. Awale $M$ and Reymond JL: Polypharmacology Browser PPB2: Target prediction combining nearest neighbors with machine learning. J Chem Inf Model 59: 10-17, 2019.

57. Duvenaud DK, Maclaurin D, Iparraguirre J, Bombarell R, Hirzel T, Aspuru-Guzik A and Adams RP: Convolutional networks on graphs for learning molecular fingerprints. In: Advances in neural information processing systems 28. NIPS Foundation, Montreal 2: 2224-2232, 2015.

58. Putin E, Asadulaev A, Ivanenkov Y, Aladinskiy V, SanchezLengeling B, Aspuru-Guzik A and Zhavoronkov A: Reinforced adversarial neural computer for de novo molecular design. J Chem Inf Model 58: 1194-1204, 2018.

59. Olivecrona M, Blaschke T, Engkvist O and Chen H: Molecular de-novo design through deep reinforcement learning. J Cheminform 9: 48, 2017.

60. Mayr A, Klambauer G, Unterithiner $\mathrm{T}$ and Hochreiter S: DeepTox: Toxicity prediction using deep learning. Front Environ Sci 3: 80, 2016

61. Steiner S, Wolf J, Glatzel S, Andreou A, Granda JM, Keenan G, Hinkley T, Aragon-Camarasa G, Kitson PJ, Angelone D and Cronin L: Organic synthesis in a modular robotic system driven by a chemical programming language. Science 363: eaav2211, 2019.

62. Coley CW, Rogers L, Green WH and Jensen KF: SCScore: Synthetic complexity learned from a reaction corpus. J Chem Inf Model 58: 252-261, 2018.

63. Antunes DA, Devaurs D and Kavraki LE: Understanding the challenges of protein flexibility in drug design. Expert Opin Drug Discov 10: 1301-1313, 2015.

64. Uehara S and Tanaka S: AutoDock-GIST: Incorporating thermodynamics of active-site water into scoring function for accurate protein-ligand docking. Molecules 21: 1604, 2016.

65. Jamal S, Ali W, Nagpal P, Grover S and Grover A: Computational models for the prediction of adverse cardiovascular drug reactions. J Transl Med 17: 171, 2019.

66. Walonoski J, Kramer M, Nichols J, Quina A, Moesel C, Hall D, Duffett C, Dube K, Gallagher T and McLachlan S: Synthea: An approach, method, and software mechanism for generating synthetic patients and the synthetic electronic health care record. J Am Med Inform Assoc 25: 230-238, 2018.

67. Choi E, Biswal S, Malin B, Duke J, Stewart WF and Sun J: Generating multi-label discrete patient records using generative adversarial networks. PMLR 68: 286-305, 2017.

68. Linardatos P, Papastefanopoulos V and Kotsiantis S: Explainable AI: A Review of Machine Learning Interpretability Methods. Entropy (Basel) 23: 18, 2021.

This work is licensed under a Creative Commons Attribution-NonCommercial-NoDerivatives 4.0 International (CC BY-NC-ND 4.0) License. 